\title{
Percepción de los padres de niños hospitalizados sobre confianza y satisfacción en el Programa Nacional de Vacunación
}

\section{Parents' perception of hospitalized children about confidence and satisfaction in the National Vaccination Program}

\author{
Mirelys Sarduy-Lugo ${ }^{1}$ *, Anabel Sarduy-Lugo ${ }^{2}$ LidiamEsther Collado-Cabañin $^{1}$ y Roberto Valentín Lara-Pérez ${ }^{3}$ \\ ${ }^{1}$ Hospital Pediátrico Universitario "José Luis Miranda", Cuba \\ ${ }^{2}$ Universidad Estatal Península Santa Elena, Ecuador \\ ${ }^{3}$ Hospital Clínico Quirúrgico Universitario Provincial “Arnaldo Milian Castro”, Cuba \\ *mirelyss1@infomed.sld.cu
}

DOI: https://doi.org/10.26871/killkana_salud.v4i2.642

\begin{abstract}
Resumen
Introducción: Aunque la cobertura de vacunación en Cuba es superior al $98 \%$ resulta importante evaluar la percepción de los padres de niños hospitalizados sobre el cumplimiento del esquema en función de detectar las debilidades que existan en ellos en relación al tema y poder trazar estrategias en la atención de salud que permitan mantener o elevar este indicador en la población pediátrica. Objetivo: Evaluar la percepción de los padres de niños hospitalizados sobre el cumplimiento del esquema de vacunación. Metodología: Se realizó un estudio descriptivo de corte transversal en el Hospital Pediátrico Universitario "José Luis Miranda" en Villa Clara, Cuba, durante marzo de 2019. El universo de estudio quedó constituido por 1000 padres de niños cubanos con 48 o más horas de ingresados en todos los servicios del hospital durante marzo de 2019 que refirieron estar dispuestos a participar en la investigación. Resultados: Prevalecieron los padres entre los grupos de edades de 20 a 27 años con 309 y de entre 28 a 35 años con 358, el sexo estuvo más representado por las mujeres con 858, el nivel escolar pre universitario con 618 y universitario con 170 fueron los más representados, los municipios que aportaron el mayor número de ingresos en el mes estudiado fueron Santa Clara con 340 y Ranchuelo con 120. El 100\% de los padres tuvieron una percepción adecuada sobre los aspectos de la vacunación infantil explorados. Conclusión: La percepción que tienen los padres de niños hospitalizados sobre la vacunación infantil es adecuada lo cual se relaciona de forma positiva con el cumplimiento del esquema de vacunación.
\end{abstract}

Palabras clave: vacunación, esquemas de inmunización, pediatría, enfermería, cobertura universal de salud, cobertura de vacunación.

\begin{abstract}
Introduction: Although vaccination coverage in Cuba is over 98\%, it is essential to evaluate the parental perception of hospitalized children about the scheme compliance to detect their weaknesses on the subject; and be able to draw up health care strategies to maintain or raise this indicator in the paediatric population. Objective: To evaluate the parents' perception of hospitalized children about vaccination schedule compliance. Methodology: A cross-sectional descriptive study took place at the "José Luis Miranda" Pediatric University Hospital in Villa Clara, Cuba, during March 2019. The study included 1000 parents of Cuban children who were hospitalized for 48 or more in the different hospital services during March 2019, who agreed to participate in the research. Results: The group of parents aged 20 to 27 years accounted for 309, those aged 28 to 35 years for 358. The female sex was the most representative with 858; the pre-university school level with 618, and the university level with 170, were the most representative. The municipalities that contributed the most income during the month studied were Santa Clara with 340 and Ranchuelo with 120. 100\% of parents had adequate insight into the explored aspects of childhood vaccination. Conclusion: The parental perception of hospitalized children about child immunization is correct, and it is positively related to the immunization schedule compliance.
\end{abstract}

Keywords: vaccination, immunization schedules, pediatrics, Nursing, universal health coverage, vaccination coverage.

\section{Introducción}

Antes de la introducción de los calendarios de vacunación en Cuba y el mundo, las enfermedades infecciosas 
eran la causa principal de mortalidad infantil y las epidemias eran frecuentes, hasta finales del siglo pasado los niños podían padecer enfermedades infecciosas graves, con múltiples secuelas y eventualmente mortales, que hoy son totalmente prevenibles. Ejemplo, es el caso de la poliomielitis, enfermedad que redujo el número de casos y muertes atribuibles drásticamente tras el inicio de la vacunación en Cuba en 1962, y desde 1994 América quedó certificada como libre de poliovirus, seguida por la eliminación en la región del Pacífico Oeste en 2000 y en Europa en 2002. 1,2

Los éxitos alcanzados en relación con la prevención de enfermedades a través de la vacunación infantil deben guardar relación directa con el esfuerzo de los estados y gobiernos; los países que han logrado instaurar políticas estatales de salud pública donde se garantizan a todos de forma gratuita y con un nivel de accesibilidad adecuado la vacunación desde la infancia deben evidenciar un avance satisfactorio en este sentido y los pueblos deben exhibir un nivel de educación y preparación superior al respecto que se traduce en porcentajes elevados de cobertura.

La extensión de las vacunas en la mayoría de los países del mundo las ha convertido en intervenciones de salud pública sistemáticas y masivas, además de una de las acciones preventivas más importantes a este nivel. ${ }^{3,4}$

A pesar de los importantes avances obtenidos en el control de las enfermedades infecciosas mediante la inmunización, la aplicación de vacunas no está libre de polémicas, ya que, al disminuir la frecuencia de las enfermedades, adquieren mayor importancia los eventos adversos que pueden aparecer después de su aplicación, lo que trae como consecuencia las dudas sobre ellas entre la población. ${ }^{4}$

Los programas de inmunización son los responsables de responder a cualquier preocupación pública relacionada con las vacunas, al tener la tarea de brindar a los trabajadores de la salud la información más actualizada además de, el fortalecimiento de los vínculos con los medios de comunicación para brindar mensajes claros y eficaces a la población. ${ }^{4}$

Para enfermería el estudio de los conocimientos, actitudes y percepciones permite valorar las capacidades de los usuarios y en función de ello, diseñar las intervenciones educativas necesarias y pertinentes para ayudarles a tomar decisiones informadas, reforzar sus capacidades de autocuidado y estimularlos a participar de mejor forma en el cuidado de su salud o de la de los suyos. ${ }^{5}$

Aunque la cobertura de vacunación en Cuba es superior al $98 \%$ resulta importante evaluar de forma frecuente la percepción de los padres sobre el cumplimiento del esquema en función de detectar debilidades que existan en ellos en relación al tema y poder trazar estrategias en la atención de salud, que permitan mantener o elevar los indicadores de vacunación en la población pediátrica, lo cual constituye el problema de investigación y justifica la realización del presente estudio.
El objetivo de la investigación fue evaluar la percepción de los padres de niños hospitalizados sobre el cumplimiento del esquema de vacunación.

\section{Métodos}

Se realizó un estudio cuantitativo con enfoque descriptivo de corte transversal en el Hospital Pediátrico Universitario Provincial "José Luis Miranda" en Villa Clara, Cuba, durante marzo de 2019.

El universo de estudio quedó constituido por 1000 padres de niños cubanos con 48 o más horas de ingresados en todos los servicios del hospital durante marzo de 2019 que refirieron estar dispuestos a participar en la investigación.

Variables:

Percepción de los padres sobre satisfacción y confianza en el esquema de vacunación

Alta: Cuando responde de forma positiva todas las preguntas respecto al tema

Media: Cuando responde de forma positiva al menos el $50 \%$ de las preguntas

Baja: Cuando no responde de forma positiva ninguna pregunta

Para caracterizar a los padres, los autores le realizaron una entrevista semiestructurada en la unidad del paciente que como promedio duró cinco minutos, donde se obtuvo información sobre las variables edad, sexo, escolaridad (grado académico más alto alcanzado) y lugar (municipio) de residencia. En el carnet de vacunación del niño se comprobó el cumplimiento del esquema de vacunación, se consideró cumplido cuando se encontró el registro de todas las vacunas que por la edad correspondían tener administrada el niño, e incumplido cuando no se encontró el registro de una o más vacunas que por la edad correspondían tener administrada el niño.

El instrumento utilizado para evaluar la percepción de los padres sobre el cumplimiento del esquema de vacunación fue una encuesta elaborada según las recomendaciones de la Organización Mundial de la Salud ${ }^{6}$ que fue validada en investigación previa $^{7}$

\subsection{Métodos de recolección de la información y análisis estadístico}

Se aplicó una encuesta diseñada y validada por Zunino y $\operatorname{coll}^{8}$ que permitió evaluar la percepción de los padres en relación a la satisfacción y confianza que tienen del esquema de vacunación.

La encuesta incluyó preguntas cerradas y se utilizó una escala semicuantitativa de Likert para evaluar las respuestas, teniéndose en cuenta negativo (1 y 2), neutro (3), positivo (4 y 5). Los valores negativos se interpretaron como una baja percepción sobre la satisfacción y confianza y los positivos como alta.

Las variables nominales y numéricas discretas se describen con frecuencias absolutas y relativas, y las continuas con media, mediana y rango. En la comparación de la 
frecuencia se utilizó la prueba de chi cuadrado. Se consideró significativo $\mathrm{p}<0,05$. Toda la información recopilada durante el proceso investigativo se llevó a una base de datos de Excel para su procesamiento estadístico en el cual se utilizó el programa STATA V.15.0.

\subsection{Aspectos éticos}

Este estudio se sustentó en la Declaración de Helsinki, en donde se establecen los criterios de respeto, confidencialidad y dignidad de las personas, que describe lo siguiente: se deberá contar con el consentimiento por escrito; por tanto, se tuvo en cuenta los principios éticos de la investigación, se respetó la voluntariedad de los padres para participar en el estudio y el consentimiento informado. Se informó previamente a los padres en qué consistía la investigación. Se obtuvo el permiso de la Comisión científica de la institución para llevar a cabo la presente investigación.

\section{Resultados}

La distribución de la muestra de estudio atendiendo a las características de los encuestados se muestra en la tabla 1, la media de edad fue de 28,6 años, la mayoría eran mujeres $(85,80 \%)$, con nivel escolar preuniversitario $(61,60 \%)$, vínculo laboral el $63,30 \%$ y de procedencia urbana el 65,00\%.

Tabla 1: Características de los encuestados, $(\mathrm{n}=1000)$

\begin{tabular}{|lc|}
\hline Edad (años) media (rango) & $28,6(18-72)$ \\
\hline Género (n, \%) & \\
Masculino & $142(14,20)$ \\
Femenino & $858(85,80)$ \\
\hline Nivel escolar (n, \%) & \\
Secundaria & $117(11,70)$ \\
Técnico medio & $97(9,70)$ \\
Pre universitario & $616(61,60)$ \\
Universitario & $170(17,00)$ \\
\hline Empleo (n, \%) & \\
Amas de casa & $258(25,80)$ \\
Estudiantes & $91(9,10)$ \\
Con vínculo laboral & $633(63,30)$ \\
Sin vínculo laboral & $18(1,80 \%)$ \\
\hline Procedencia (n, \%) & \\
Urbana & $650(65,00)$ \\
Rural & $340(34,00)$ \\
\hline
\end{tabular}

Fuente: Encuesta

En la tabla 2 se describe la percepción de los padres sobre confianza en el Programa Nacional de Vacunación a través del grado de acuerdo con la importancia de las vacunas. La confianza general en las vacunas fue alta en $989(98,90 \%)$ y media en $11(1,10 \%)$. La valoración de la seguridad en las vacunas fue alta en $990(99,00 \%)$ y media en $10(1,00 \%)$. El $100 \%$ de los encuestados considera importante la vacunación para prevenir enfermedades en niños y adultos, $(\mathrm{p}<0,01)$.

Tabla 2: Percepción de los padres de niños hospitalizados sobre el nivel de confianza en el Programa Nacional de Vacunación, Santa Clara, $2019(\mathrm{n}=1000)$

\begin{tabular}{lcc}
\hline \multicolumn{1}{c}{ Variable/Nivel de confianza } & Medio & Alto \\
\hline $\begin{array}{l}\text { Le parece correcto vacunar a sus hijos } \\
\text { Le parece importante la vacunación de los niños para } \\
\text { prevenir enfermedades. }\end{array}$ & $0(0,00 \%)$ & $11(1,1000(100,00 \%)$ \\
$\begin{array}{l}\text { Le parece importante la vacunación de los niños para } \\
\text { evitar el contagio }\end{array}$ & $11(1,10 \%)$ & $989(98,90 \%)$ \\
$\begin{array}{l}\text { Le parece importante la vacunación de los adultos para } \\
\text { prevenir enfermedades. }\end{array}$ & $0(0,00 \%)$ & $1000(100,00 \%)$ \\
$\begin{array}{l}\text { Le parece importante la vacunación de los adultos para } \\
\text { evitar el contagio }\end{array}$ & $11(1,10 \%)$ & $989(98,90 \%)$ \\
\hline
\end{tabular}

Fuente: Encuesta

El nivel de satisfacción con el Programa nacional de Vacunación fue alto. En la tabla 3 se describe las diferentes categorías comprendidas en esta variable. El $100 \%$ de los encuestados coincidieron en que el funcionamiento del vacunatorio es adecuado y mostraron satisfacción con las vacunas incluidas en el esquema vigente; con la información brindada por el Ministerio de Salud 989 personas $(98,90 \%)$, y por el médico y la enfermera de la Atención Primaria de Salud (APS) el $100 \%$.

Tabla 3: Percepción de los padres de niños hospitalizados sobre el nivel de satisfacción con el Programa nacional de Vacunación, Santa Clara, $2019(n=1000)$

\begin{tabular}{lcc}
\hline \multicolumn{1}{c}{ Variable/Nivel de satisfacción } & Medio & Alto \\
\hline Funcionamiento del vacunatorio & $0(0,00 \%)$ & $1000(100,00 \%)$ \\
Tipo de vacunas incluidas & $0(0,00 \%)$ & $1000(100,00 \%)$ \\
Información brindada por el Ministerio de Salud & $11(1,10 \%)$ & $989(98,90 \%)$ \\
Información brindada por el médico y la enfermera de la Atención & $0(0,00 \%)$ & $1000(100,00 \%)$ \\
Primaria de la Salud & & \\
\hline \multicolumn{3}{c}{ Fuente: Encuesta } \\
\end{tabular}

Referente al medio que utilizan para mantenerse informado sobre las actividades relacionadas con la vacunación fue referido en primer lugar el médico y la enfermera del área de salud por 996 personas $(99,60 \%)$, la televisión nacional por 998 encuestados $(99,80 \%)$, la prensa escrita en 990 personas $(99,00 \%)$ y la radio en 994 encuestados $(99,40 \%)$.

La relación entre el cumplimiento del esquema de vacunación evaluado por auditorías a los carnet de vacunación de los niños y la relación con la percepción de los padres sobre satisfacción y confianza en el Programa Nacional de Vacunación se muestra en la tabla 4 donde el $100 \%$ de los padres encuestados tenían una percepción adecuada en relación con la seguridad y confianza, mientras que 989 carnet de vacunación auditados mostraron el esquema cumplido hasta el momento del estudio $(\mathrm{p}<0,01)$. Lo que demuestra una relación directa entre el pensamiento de los padres sobre vacunación y la práctica llevada a hecho en sus hijos. 
Tabla 4: Distribución porcentual de padres según cumplimiento del esquema de vacunación y percepción sobre vacunación

\begin{tabular}{|c|c|c|c|c|c|c|}
\hline \multirow{3}{*}{ Cumplimiento } & \multicolumn{4}{|c|}{ Percepción } & \multirow{2}{*}{\multicolumn{2}{|c|}{ Total }} \\
\hline & \multicolumn{2}{|c|}{ Adecuada } & \multicolumn{2}{|c|}{ Inadecuada } & & \\
\hline & No & $\%$ & No & $\%$ & No & $\%$ \\
\hline Cumplido & 989 & 98,90 & 0 & 0,00 & 989 & 98,90 \\
\hline Incumplido & 11 & 1,10 & 0 & 0,00 & 11 & 1,10 \\
\hline Total & 1000 & 100,00 & 0 & 0,00 & 1000 & 100,00 \\
\hline
\end{tabular}

Fuente: Base de datos

\section{Discusión}

Es criterio de las autoras que los resultados encontrados en relación con el sexo prevalente en la muestra estudiada guarda relación con la idiosincrasia de la sociedad cubana donde a pesar de tener los mismos derechos tanto hombres como mujeres, el cuidado directo de los hijos sobre todo los enfermos recaen en la mujer.

Según criterio de las autoras el nivel escolar de los padres puede ser un elemento que influye en la percepción de estos sobre vacunación y en los conocimientos o preparación que estos tengan sobre este tema, a pesar que los resultados obtenidos en el estudio no mostraron significancia estadística en este sentido, similares resultados encontró Zunino y coll en un estudio realizado en Montevideo, Uruguay. ${ }^{7}$

Más de un artículo refleja la importancia de que tanto las autoridades como los profesionales sanitarios no descuiden la necesidad de mejorar los conocimientos de la población en este tema, haciendo difusión activa y de forma accesible de documentación y materiales con contenidos veraces, rigurosos e inteligibles y dando respuesta las dudas que pacientes y familias puedan plantear. ${ }^{8,9}$

Investigaciones reflejan la realidad actual sobre el tema de la resistencia a las vacunas en el mundo, sobre todo en individuos de renta y escolaridad alta, ${ }^{8}$ lo cual se ha convertido en un asunto notable para la salud pública en varios países. $^{10,11}$

Según criterio de las autoras este fenómeno de la resistencia a las vacunas puede verse reflejado primeramente por la disminución de la cobertura vacunal en lugares donde se alcanzó en algún momento índices elevados en este sentido, lo cual debe ser vigilado con extrema frecuencia para poder advertir de esta situación a tiempo y poder controlarla de forma adecuada.

La segunda forma en que puede verse reflejado este problema es con el resurgimiento de enfermedades ya controladas, lo cual es la consecuencia final de no haber tratado de forma adecuada la situación deficiente de cobertura. ${ }^{12}$

Los movimientos antivacunas creados y extendidos en algunos países han generado dudas en la población sobre la eficiencia y eficacia de la vacunación, lo cual unido a la deficiente información que sobre este tema mantienen los trabajadores sanitarios han perpetuado la disminución brusca y preocupante de la cobertura vacunal sustentado sobre todo por una percepción inadecuada de los padres sobre esta medida preventiva tan importante y una erosión de la confianza pública en las instituciones involucradas en la vacunación. ${ }^{13,14}$

Las dudas fundamentales sobre vacunación en la población son en relación al uso de adyuvantes en los preparados vacunales, a los cuales se le atribuye ser causa de efectos adversos por su toxicidad. ${ }^{15-17}$

En Cuba este fenómeno aún no se ha experimentado gracias a las políticas de salud que garantizan primero una información adecuada de la población lo cual genera una cobertura vacunal superior al $98 \%$, también son reflejos de estos logros el hecho de que la salud en Cuba es pública, gratuita y accesible, unido a que el Programa Atención Materno Infantil y la Atención Primaria de Salud mantienen un control estricto del cumplimiento del esquema de vacunación en cada niño.

Cuando se compara los niveles de cobertura de Cuba en relación a la vacunación con el de otros países se observa diferencias notorias, por ejemplo, en países de la Unión Europea (UE) este indicador se encuentra entre 80-90\%, lo cual demuestra que a pesar de que el nivel económico y de desarrollo de estos países supera al de la nación caribeña, esta ha logrado crear estrategias que le han garantizado alcanzar una cobertura superior al $98 \% .^{18,19}$

\section{Conclusiones}

La satisfacción y confianza en el Programa Nacional de vacunación es alta. La percepción que tienen los padres de niños hospitalizados sobre la vacunación infantil es adecuada lo cual se relaciona de forma positiva con el cumplimiento del esquema de vacunación.

\section{Fuente de Financiamiento}

Este estudio es autofinanciado.

\section{Conflicto de Intereses}

No existen conflictos personales, profesionales, financieroso de otro tipo.

\section{Consentimiento Informado}

Los autores cuentan con el consentimiento informado de los pacientes para la investigación, la publicación del caso y sus imágenes.

\section{Referencias Bibliográficas}

1. Izaguirre González AI, Zerón Moreno J. Parotiditis reemergente: un nuevo brote epidémico. Rev méd hondur. 2018:64-70.

2. Chaple EB. Una batalla ganada: la eliminación de la poliomielitis en Cuba. História, Ciências, Saúde-Manguinhos. 2015;22(3):961-983.

3. Cruz Piqueras M, Rodríguez García de Cortazar A, Hortal Carmona J, Padilla Bernáldez J. Reticencia vacunal: análisis del discurso de madres y padres con rechazo total o parcial a las vacunas. Gaceta sanitaria. 2019;33:53-59. 
4. Mejía Salas H. VACUNACIóN EN GRUPOS ESPECIALES. Revista Médica La Paz. 2016;22(2):98-103.

5. Véliz L, Campos C, Vega P. Conocimiento y actitudes de los padres en relación a la vacunación de sus hijos. Revista chilena de infectología. 2016;33(1):30-37.

6. Organización Panamericana de la Salud/Organización Mundial de la Salud. La semana de vacunación en las Américas 2016, 23-30 de abril. Cuaderno de planificación y evaluación. Washington DC: OPS;. Available from: 1 http://www.paho.org/vwa/wp-content/uploads/2016/03/ CUADERNO-DE-PLANIFICACION-SVA-2016.docx.

7. Zunino C, Speranza N, González V, Silva P, Varela A, Giachetto $\mathrm{G}$, et al. Evaluación de la confianza y satisfacción 17. del Programa Nacional de Vacunaciones en Montevideo, Uruguay, entre mayo y octubre de 2016. Archivos de Pediatría del Uruguay. 2019;90(2):69-77.

8. Le-Corre N, Barría S, López T, Martínez-Valdebenito C, 18. Contreras AM, Ferrés M. Parotiditis en Chile: caracterización clínica y molecular de dos casos en una población altamente inmunizada. Revista chilena de infectología. 2018;35(2):198-203.

9. de Patología Infecciosa PG. Posicionamiento de la 19. Asociación Española de Pediatría de Atención Primaria (AEPap) sobre la vacunación infantil: análisis crítico y propuestas de mejora (enero de 2016). Revista Pediatría de Atención Primaria. 2016;18(69):17.

10. Iriart JAB. Autonomía individual vs. protección colectiva: la no vacunación infantil entre las capas de mayor renta/escolaridad como desafío para la salud pública. SciELO Public Health; 2017.

11. Kang HJ, Kim SH, Chung JK, Lee SW, Choi SB, Eom HE, et al. Viral etiology of sporadic cases of parotitis among children in Korea during 2013-2014. Journal of Medical Virology. 2018;90(1):61-66.

12. Berezin M, Eads A. Risk Is for the Rich? Childhood Vaccination Resistance and a Culture of Health Soc Sci Med 1982 2016; 165: 233-45;

13. de Souza EJ, Beltrame V, Junior BRS, Turra L. Atitudes de pais não vacinadores podem ser uma ameaça ao Brasil? Anais de Medicina. 2017.

14. Ponce-Blandón JA, Díaz-Ruiz M, Pabón-Carrasco M, Lomas-Campos M. EL MOVIMIENTO ANTI-
VACUNAS COMO PROBLEMA DE SALUD PÚBLICA: UNA REVISIÓN INTEGRATIVA DE LA LITERATURA. Revista de Enfermagem da UFSM. 2018 Dec;8(4):812-828.

15. Arellán-Regalado M. Conocimientos y actitudes de madres con hijos menores de 5 años sobre vacunas. CASUS Revista de Investigación y Casos en Salud. 2018;3(3):130-137.

16. Albi Rodríguez M, Aparicio Rodrigo M. Sin datos a largo plazo, las vacunas con nuevos adyuvantes aumentan discretamente los efectos adversos a corto y medio plazo. Evid Pediatr. 2016;12(42):4.

. Stassijns J, Bollaerts K, Baay M, Verstraeten T. A systematic review and meta-analysis on the safety of newly adjuvanted vaccines among children. Vaccine. 2016;34(6):714-722.

8. Hernández-Sampelayo Matos T, Merino Moína M, Ortigosa del Castillo L, Ruiz-Contreras J. Seguridad de las vacunas utilizadas en niños. ¿ Los nuevos adyuvantes pueden alterar el equilibrio entre eficacia y toxicidad? 2005.

9. Méndez-Herrera A, Arias-Toledo E, Sánchez-Alemán M, Olamendi-Portugal M, Ruiz-Gómez J, Díaz-Ortega JL, et al. Prevalencia de anticuerpos frente a parotiditis en niños y adolescentes a dos años de haberse introducido la vacuna SRP. salud pública de méxico. 2018;60:71-76.

Recibido: 3 de febrero de 2020

Aceptado: 5 de julio de 2020 
